\title{
Sulforaphane protects against 6-hydroxydopamine-induced cytotoxicity by increasing expression of heme oxygenase-1 in a PI3K/Akt-dependent manner
}

\author{
CHANG DENG ${ }^{1}$, RAN TAO $^{2}$, SHUN-ZHI YU ${ }^{3}$ and HUA JIN ${ }^{4}$ \\ ${ }^{1}$ Department of Emergency, The People's Hospital of Guang Du District, Kunming 650200; ${ }^{2}$ Second Department \\ of Internal Medicine, The People's Hospital of Panlong District, Kunming 650021; ${ }^{3}$ Mental Health Hospital \\ of Prevention and Treatment in Kunming City, Kunming 650041; ${ }^{4}$ Department of Anesthesia, \\ The First People's Hospital of Yunnan Province, Kunming 650032, P.R. China
}

Received October 4, 2011; Accepted December 19, 2011

DOI: $10.3892 / \mathrm{mmr} .2011 .731$

\begin{abstract}
Parkinson's disease (PD) is a progressive neurodegenerative disorder with selective loss of dopaminergic neurons in the substantia nigra. Evidence suggests that oxidative stress is involved in the pathogenesis of PD. Sulforaphane (SF), a naturally occurring isothiocyanate, has been shown to protect against oxidative stress by inducing the expression of various NF-E2-related factor-2 (Nrf2) responsive genes. Previous studies have shown that SF protects dopaminergic neurons against PD-related neurotoxin 6-hydroxydopamine (6-OHDA)-induced cytotoxicity. However, the molecular mechanisms by which SF protects against 6-OHDA-induced cytotoxicity are poorly elucidated. In this study, we found that pretreatment with SF significantly reduced 6-OHDAinduced caspase-3 activation and subsequent cell death. SF also increased heme oxygenase-1 (HO-1) expression, which conferred protection against 6-OHDA-induced cytotoxicity. Furthermore, SF induced the translocation of Nrf2 into the nucleus and activated PI3K/Akt, a pathway that is involved in SF-induced Nrf2 nuclear translocation, HO-1 expression and cytoprotection. These results suggest that SF inhibits 6-OHDA-induced cytotoxicity through increasing HO-1 expression in a PI3K/Akt-dependent manner.
\end{abstract}

\section{Introduction}

Parkinson's disease (PD) is a common neurodegenerative disorder characterized by progressive degeneration and loss of dopaminergic neurons in the substantia nigra pars compacta

Correspondence to: Dr Hua Jin, Department of Anesthesia, The First People's Hospital of Yunnan Province, Kunming 650032, P.R. China

E-mail: jinghuakm@163.com

Key words: sulforaphane, 6-hydroxydopamine, heme oxygenase-1, Parkinson's disease and dopaminergic nerve terminals in the striatum (1). Although the etiology of PD remains unclear, previous studies have shown that oxidative stress is involved in the pathogenesis of PD (2,3). 6-Hydroxydopamine (6-OHDA) is commonly used to produce in vivo and in vitro models of PD $(4,5)$. 6-OHDA can be formed from dopamine by non-enzymatic hydroxylation in the presence of $\mathrm{Fe}^{2+}$ and $\mathrm{H}_{2} \mathrm{O}_{2}$ (6,7). 6-OHDA stimulates the production of superoxide radicals and induces cell death (8). Numerous experiments have revealed that antioxidants exert beneficial effects in the prevention of 6-OHDA-induced cytotoxicity $(9,10)$.

Heme oxygenase-1 (HO-1) is an enzyme that catalyzes the degradation of heme. It has been widely reported that $\mathrm{HO}-1$ plays a crucial role in maintaining redox homeostasis during cellular stress (11-13). Increased HO-1 expression contributes to promoting the degradation of heme into carbon monoxide and biliverdin, the latter being the immediate precursor of the powerful antioxidant molecule bilirubin (14). The induction of HO-1 expression is primarily regulated by NF-E2-related factor 2 (Nrf2), a central transcription factor that interacts with the antioxidant response element (ARE) to activate the transcription of numerous detoxifying and antioxidant genes $(11,15)$. Under normal conditions, Nrf2 is sequestered in the cytoplasm by its actin-bound negative regulator, Keap1. However, Nrf2 can be liberated from the Keap1-Nrf2 complex and translocates into the nucleus by various stimuli, such as electrophiles and oxidative stress (16). Recently, several studies have indicated that the translocation of Nrf2 into the nucleus requires the activation of several signal transduction pathways, including the phosphatidylinositol 3-kinase (PI3K) pathway $(17,18)$. Increased evidence indicates that HO-1 provides protection against oxidative stress, and modulation of HO-1 expression may represent a novel target for therapeutic intervention.

Sulforaphane (SF) is a natural drug derived from isothiocyanate found in cruciferous vegetables such as broccoli, cabbage and cauliflower. SF has been used as a chemopreventive compound (19). The cytoprotective effect of SF is explained mainly by activation of the Nrf2-ARE pathway (20). In vivo studies have shown that SF protects the brain against hypoxic- 
ischemic injury through induction of Nrf2-dependent phase 2 enzyme (21). SF also has been reported to be a potential candidate for development of treatment and/or prevention of PD (22). SF protects dopaminergic neurons against 6-OHDA-induced cytotoxicity $(23,24)$. However, the molecular mechanisms by which SF protects against 6-OHDA-induced cytotoxicity are poorly elucidated. In the present study, we demonstrated that SF-induced activation of PI3K/Akt increases the expression of $\mathrm{HO}-1$, which in turn provides protection against 6-OHDAinduced cytotoxicity in PC12 cells.

\section{Materials and methods}

Materials. 6-Hydroxydopamine, sulforaphane, zinc protoporphyrin (znPP) and the MTT-based colorimetric assay kit were obtained from Sigma Chemical Co. (St. Louis, MO, USA). Antibodies against phosphor-Akt, Akt, $\beta$-actin, lamin B, Nrf2 and HO-1 were purchased from Santa Cruz Biotechnology (Santa Cruz, CA). RPMI-1640 medium, horse serum and fetal calf serum were obtained from Gibco-BRL/Life Technologies (Grand Island, NY, USA). Caspase fluorogenic substrate for caspase-3 (Ac-DEVD-AMC) was purchased from Alexis Biochemicals (Lausanne, Switzerland). All other chemicals were reagent grade.

Cell culture and cell viability assay. PC12 cells were cultured in RPMI-1640 medium supplemented with $10 \%$ heatinactivated horse serum, 5\% heat-inactivated fetal calf serum, $100 \mathrm{IU} / \mathrm{ml}$ penicillin and $100 \mu \mathrm{g} / \mathrm{ml}$ streptomycin at $37^{\circ} \mathrm{C}$ in a humid atmosphere of $5 \% \mathrm{CO}_{2}$. Cell viability was measured using MTT-based assays according to the manufacturer's instructions.

Determination of caspase-3 activity. The method described by Movsesyan et al was used to examine caspase-3 activity. PC12 cells were washed with PBS and extracted in lysis buffer [10 mM HEPES (pH 7.4); $50 \mathrm{mM} \mathrm{NaCl}, 2 \mathrm{mM} \mathrm{MgCl}, 5 \mathrm{mM}$ EGTA, $1 \mathrm{mM}$ phenylmethylsulfonyl fluoride and $20 \mu \mathrm{g} / \mathrm{mol} / 1$ leupeptin]. The supernatants were incubated with protease assay buffer and Ac-DEVD-AMC. The change in absorbance was measured using a spectrofluorometer.

Nuclear and cytosolic lysate preparation. Nuclear and cytosolic extracts were isolated as described previously (Huang Yuan Li, 2007). Cells were incubated in ice-cold buffer A. After 15 min of incubation on ice, NP-40 was added to a final concentration of $0.6 \%$, and then cells were vortexed and centrifuged for $1 \mathrm{~min}$ at $16,000 \mathrm{x}$. The supernatant was kept as the cytoplasmic extract. The nuclear pellet was extracted with buffer B (20 mM HEPES, pH 8.0, 1 mM EDTA, $1.5 \mathrm{mM}$ $\mathrm{MgCl}_{2}, 10 \mathrm{mM} \mathrm{KCl}, 1 \mathrm{mM}$ DTT, $1 \mathrm{mM}$ sodium orthovanadate, $1 \mathrm{mM}$ NaF, $1 \mathrm{mM}$ PMSF, $0.5 \mathrm{mg} / \mathrm{ml}$ benzamidine, $0.1 \mathrm{mg} / \mathrm{ml}$ leupeptin, $1.2 \mathrm{mg} / \mathrm{ml}$ aprotinin and $20 \%$ glycerol) for $30 \mathrm{~min}$ on ice. The debris was removed by centrifugation at $16,000 \mathrm{xg}$ for $20 \mathrm{~min}$ at $4^{\circ} \mathrm{C}$.

Western blot analysis. After treatment, cells were collected and washed twice with ice-cold phosphate-buffered saline (PBS). The harvested cells were then lysed with a solubilizing solution [20 mM Tris- $\mathrm{HCl}$ (pH 7.0), $25 \mathrm{mM} \beta$-glycerophosphate,
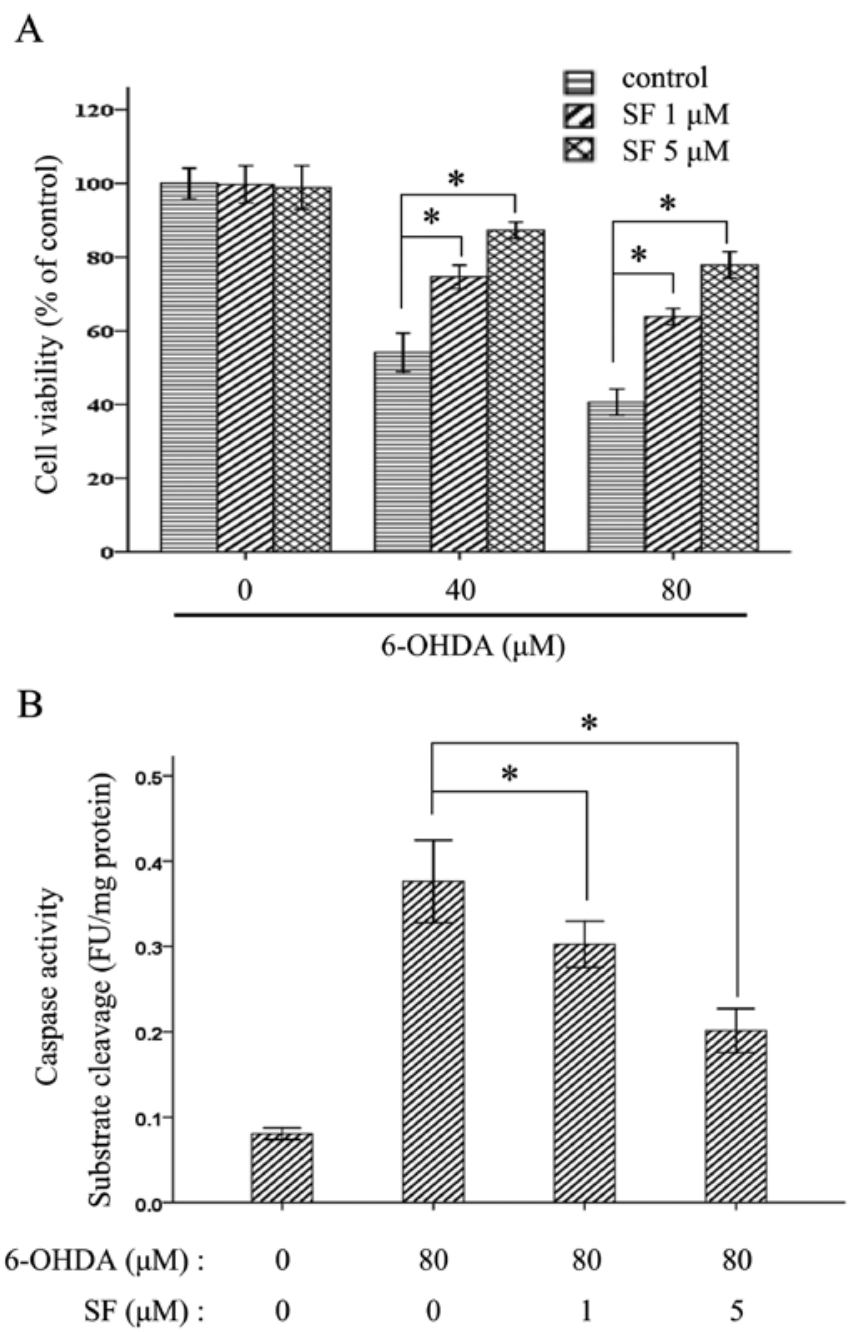

Figure 1. SF prevents 6-OHDA-induced cytotoxicity. PC12 cells were treated with SF (1 and $5 \mu \mathrm{M}$ ) for $24 \mathrm{~h}$ and then incubated with 6-OHDA (40 or $80 \mu \mathrm{M}$ ) for a further $24 \mathrm{~h}$. (A) Cell viability was measured with an MTT assay. "Statistical significance $(\mathrm{P}<0.05)$. (B) PC12 cells were treated as indicated. The catalytic activity of caspase- 3 in cell lysates was assayed using the synthetic peptide substrate Ac-DEVD-AMC. Values are means \pm SD from three separate cultures. "Significantly different from 6-OHDA-treated cells $(\mathrm{P}<0.05)$

2 mM EGTA, $1 \%$ Triton X-100, $1 \mathrm{mM}$ vanadate, $1 \%$ aprotinin, $1 \mathrm{mM}$ phenylmethylsulfonyl fluoride and $2 \mathrm{mM}$ dithiothreitol] on ice for $40 \mathrm{~min}$. The lysate was centrifuged at $15,000 \mathrm{rpm}$ for $15 \mathrm{~min}$. Supernatants were collected and protein concentrations were determined using Bio-Rad protein assay reagent (Hercules, CA, USA). An equal quantity of proteins was separated by $10 \%$ SDS-polyacrylamide gel electrophoresis and transferred to a PVDF membrane (Millipore Corp., Billerica, MA, USA). The membranes were incubated with rabbit polyclonal Akt, phospho-Akt, Nrf2, HO-1 or mouse monoclonal $\beta$-actin antibodies, washed and then incubated with peroxidase-conjugated anti-rabbit or anti-mouse $\operatorname{IgG}$ (KPL, Gaithersburg MD, USA). The immunoblot was revealed with an ECL western blot detection kit (Amersham Pharmacia Biotech, Buckinghamshire, UK).

Statistical analysis. All values are expressed as means \pm SD. Data were analyzed by ANOVA followed by Tukey-Kramer 
A

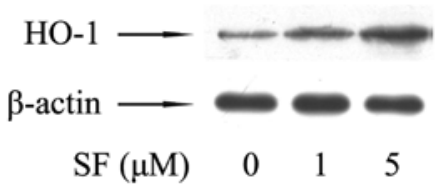

B

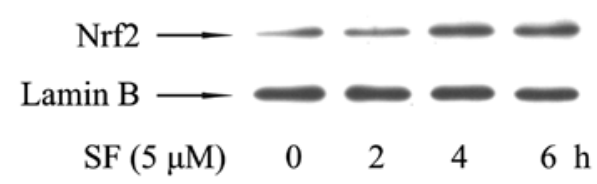

Figure 2. Effects of SF on HO-1 expression and Nrf2 translocation into the nucleus. (A) PC12 cells were exposed to SF (1 and $5 \mu \mathrm{M}$ ) for $24 \mathrm{~h}$. HO-1 expression was measured by western blot analysis. All experiments were conducted in triplicate. (B) PC12 cells were treated with SF $(5 \mu \mathrm{M})$ for the indicated times and nuclear extracts were prepared for western blotting. Nrf2 expression was assessed. All experiments were conducted in triplicate.

test as the post hoc test. Differences were considered statistically significant at a level of $\mathrm{P}<0.05$.

\section{Results}

SF protects against 6-OHDA-induced cytotoxicity. To determine the effect of SF on 6-OHDA-induced cytotoxicity, we exposed PC12 cells to SF for $24 \mathrm{~h}$ prior to exposure to 6-OHDA for a further $24 \mathrm{~h}$. As shown in Fig. 1A, pretreatment with SF protected cells against 6-OHDA-induced cell death in a dose-dependent manner. It has been previously demonstrated that 6-OHDA induces the activation of caspase-3, which plays an important role in the terminal execution phase of apoptosis. In the present study, we found that 6-OHDA-induced caspase-3 activation was suppressed by the pretreatment with SF (Fig. 1B). These results indicate that SF inhibits 6-OHDAinduced apoptosis in PC12 cells.

SF induces the translocation of Nrf2 into the nucleus and increases $\mathrm{HO}-1$ expression. Previous studies have demonstrated that $\mathrm{SF}$ is a potent Nrf2 activator and exhibits antioxidative effects via the up-regulation of ARE-driven genes. To investigate the neuroprotective mechanisms of SF, we examined the effect of SF on HO-1 expression and the translocation of Nrf2 into the nucleus in PC12 cells. As shown in Fig. 2B, SF increased the amount of Nrf2 after $2 \mathrm{~h}$ in the nuclear fraction. In this study, we also found that the expression of $\mathrm{HO}-1$, an important ARE-driven gene, was increased by SF in a dosedependent manner (Fig. 2A).

Involvement of the PI3K/Akt pathway in $\mathrm{HO}-1$ expression by $S F$. It has been widely reported that the PI3K/Akt pathway is involved in regulating the activation of the Nrf2-ARE pathway. To determine the upstream signaling pathway involved in SF-mediated Nrf2 activation and induction of HO-1, we examined whether SF activates the PI3K/Akt pathway. As shown in Fig. 3A, SF phosphorylated Akt in PC12 cells (Fig. 3A). Furthermore, HO-1 expression and nuclear accumulation of Nrf2 were reduced by the PI3K inhibitor LY294002 (Fig. 3B and $\mathrm{C}$ ). These results suggest that PI3K/Akt signaling plays a
A

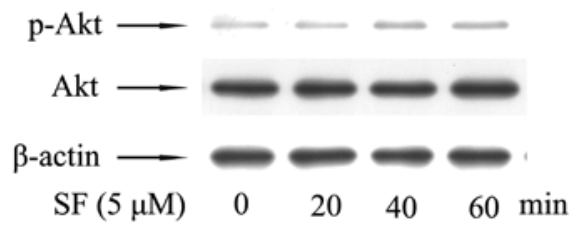

B

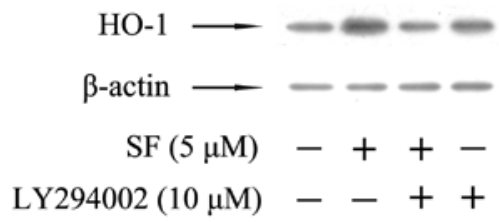

$\mathrm{C}$

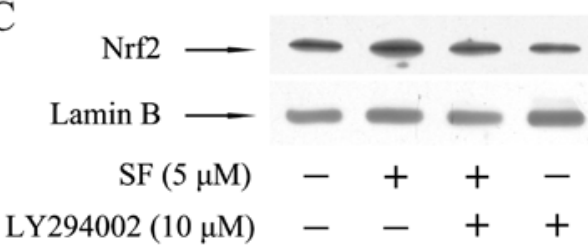

Figure 3. Induction of HO-1 and activation of Nrf2 by SF via phosphorylation. (A) Effect of SF on the phosphorylation of Akt. PC12 cells were treated with $5 \mu \mathrm{M} \mathrm{SF}$ for the indicated times. The level of $\mathrm{p}$-Akt was measured using western blot analysis. (B) Effect of PI3K inhibitor on SF-induced HO-1 expression. PC12 cells were preincubated with $20 \mu \mathrm{M}$ LY294002 for $30 \mathrm{~min}$ and then treated with $5 \mu \mathrm{M} \mathrm{SF}$ for $24 \mathrm{~h}$. Cell lysates were subjected to western blot analysis using anti-HO- 1 and anti- $\beta$-actin antibodies. (C) Effect of PI3K inhibitor on SF-induced Nrf2 translocation. PC12 cells were preincubated with $10 \mu \mathrm{M}$ LY294002 for $30 \mathrm{~min}$ and then treated with $5 \mu \mathrm{M} \mathrm{SF}$ for $4 \mathrm{~h}$. Nuclear extracts were subjected to western blot analysis by using anti-Nrf2 and anti-lamin B antibodies.

role in SF-mediated HO-1 expression through the translocation of Nrf2 into the nucleus.

PI3K/Akt pathway regulates $S F$-mediated protection against 6-OHDA. HO-1 plays an important role in the cellular defense against oxidative stress. Accordingly, we examined whether $\mathrm{HO}-1$ induction is involved in SF-mediated protection against 6-OHDA-induced cytotoxicity. As shown in Fig. 4A, the protective effect of SF on 6-OHDA-induced cytotoxicity was reversed by zinc protoporphyrin (an inhibitor of HO-1). To examine the effects of PI3K/Akt on SF-mediated protection against 6-OHDA, cells were pretreated with LY194002 for $30 \mathrm{~min}$ prior to addition of SF. The MTT assays showed that LY194002 reversed the protective effects of SF against 6-OHDA-induced cell death (Fig. 4B). Furthermore, the inhibition of 6-OHDA-induced caspase-3 activation by SF was also reversed by the pretreatment with LY194002 (Fig. 4C).

\section{Discussion}

The cytoprotective role of SF has been widely studied in vivo and in vitro. SF protects immature hippocampal neurons against death caused by exposure to hemin or to oxygen and glucose-deprivation (25). SF induces thioredoxin expression and attenuates retinal light damage in mice (26). It also has been reported that SF protects against 6-OHDA-induced cytotoxicity. However, the molecular mechanisms by which 
A
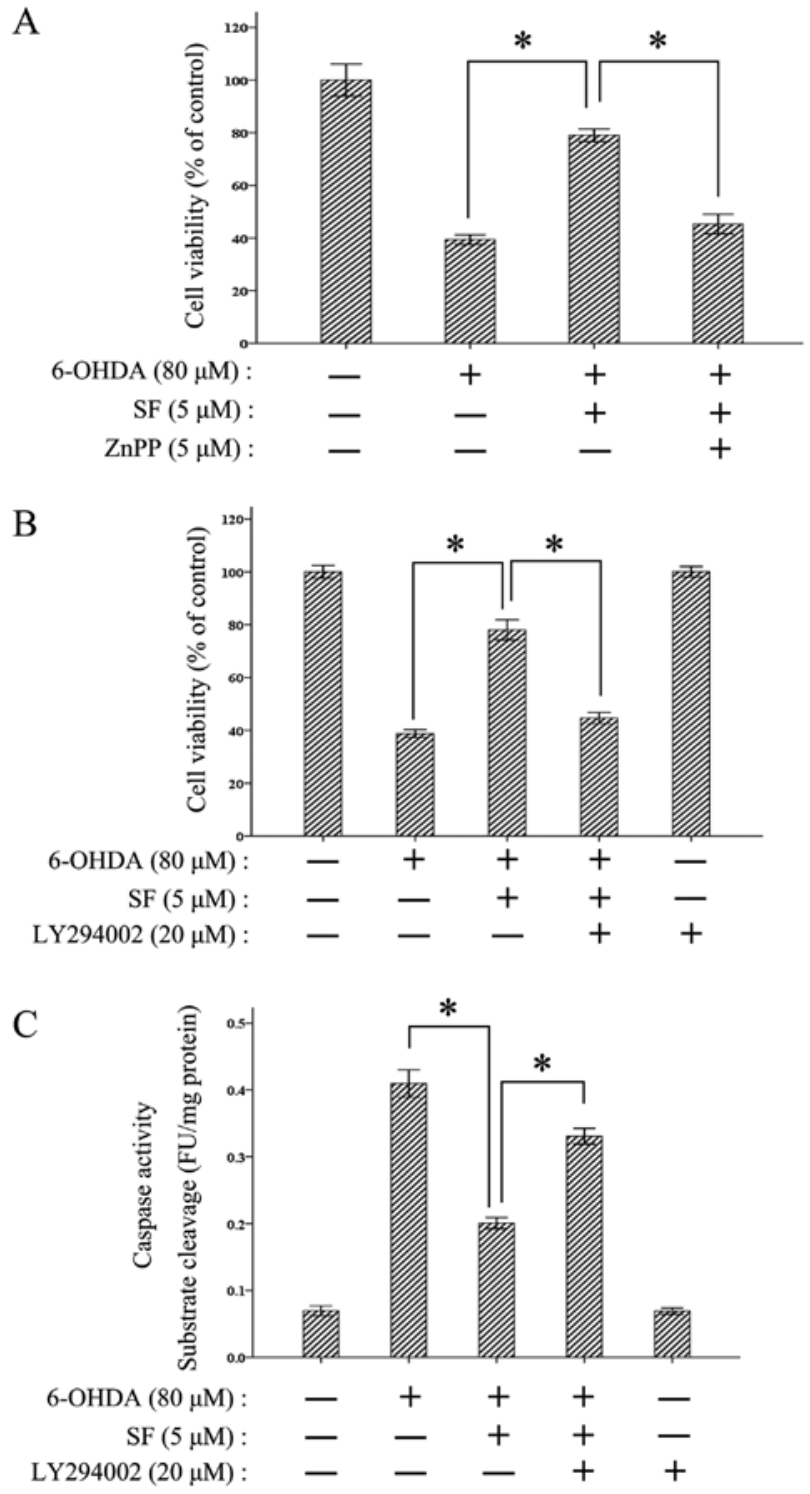

Figure 4. HO-1 and PI3K inhibitors attenuate the cytoprotective effect of SF. (A) HO-1 enzyme inhibitor $\mathrm{ZnPP}$ suppressed the protective effect of SF against 6-OHDA-induced cytotoxicity. PC12 cells were treated with $5 \mu \mathrm{M}$ $\mathrm{SF}$ for $24 \mathrm{~h}$ and then incubated with $\mathrm{ZnPP}(5 \mu \mathrm{M})$ for $30 \mathrm{~min}$ and addition of 6-OHDA $(80 \mu \mathrm{M})$ into the cells for a further $24 \mathrm{~h}$. Cell viability was measured by MTT assay. (B) PC12 cells were pretreated with LY294002 for $30 \mathrm{~min}$ and then incubated with SF. After $24 \mathrm{~h}$ of incubation with SF, cells were treated with 6-OHDA $(80 \mu \mathrm{M})$ for a further $24 \mathrm{~h}$. Cell viability was measured with an MTT assay. (C) The catalytic activity of caspase-3 in cell lysates was assayed using the synthetic peptide substrate Ac-DEVD-AMC. *Statistical significance $(\mathrm{P}<0.05)$.

SF protects against 6-OHDA-induced cytotoxicity are poorly elucidated. In the present study, we demonstrated that SF modulates HO-1 induction, Nrf2 nuclear translocation and 6-OHDA-induced cytotoxicity through the PI3K/Akt pathway. To our knowledge, this is the first report demonstrating that SF protects against 6-OHDA-induced cytotoxicity through induction of HO-1 expression in a PI3K-dependent manner.

Excessive free radical formation can result in oxidative stress, a possible mechanism of PD. Mammalian cells have developed several protective mechanisms to prevent oxidative stress (27). These mechanisms employ antioxidants to augment the oxidative defense capacity (28). It has been widely demonstrated that the Nrf2-ARE pathway plays an important role in cellular defense mechanisms (29). Nrf2 binds to the ARE in the promoter region of a number of genes, encoding for phase II detoxifying enzymes and antioxidative proteins, including thioredoxin, $\mathrm{HO}-1, \mathrm{NAD}(\mathrm{P}) \mathrm{H}$ :quinine oxidoreductase-1, glutathione reductase and glutathione peroxidase $(30,31)$. SF is a known activator of the Nrf2-ARE pathway. It has been reported that SF protects against 6-OHDA-induced oxidative stress by increasing the levels of GSH, NAD $(\mathrm{P}) \mathrm{H}$ : quinine oxidoreductase-1, GSH-transferase and reductase (23). Whether HO-1 is involved in the SF-mediated protection against 6-OHDA is still unknown. The induction of HO-1 has been considered an adaptive and benefical response to oxidative stress $(11,13)$. Overexpression of HO-1 in transgenic mice attenuates cerebral ischemic injury and $\mathrm{H}_{2} \mathrm{O}_{2}$ - or glutamate-induced oxidative stress $(29,32)$. Several studies have demonstrated that SF markedly increases HO-1 expression in liver tissues and hippocampal neurons $(25,33)$. In agreement with other studies, the induction of HO-1 in SF-treated PC12 cells has been demonstrated in this study. In addiction, we showed that $\mathrm{ZnPP}$, an inhibitor of HO-1 activity, can partially reverse the protective effects of SF. These results suggest that the increase in HO-1 expression by SF conferred cytoprotection against 6-OHDA-induced cytotoxicity.

Activation of the PI3K/Akt pathway is a crucial step in various biological processes, such as growth, survival, antiapoptosis and cell proliferation. It has been reported that HO-1 expression is regulated by activation of Nrf2 in a PI3K/ Akt-dependent manner $(17,34,35)$. In this study, we found that SF activates the PI3K/Akt pathway. The pretreatment of LY294002, an inhibitor of the PI3K/Akt pathway, suppressed SF-induced increase in HO-1 expression in PC12 cells. Also, the PI3K/Akt inhibitor blocked SF-induced Nrf2 nuclear translocation. Furthermore, the protective effects of SF against 6-OHDA-induced cytotoxicity were blocked by the PI3K/Akt inhibitor. These results suggest that the PI3K/Akt pathway is important for SF-induced Nrf2 nuclear translocation, HO-1 expression and protection.

In summary, SF induces HO-1 expression in PC12 cells, and this expression confers protection against 6-OHDAinduced cytotoxicity. SF also induces the activation of $\mathrm{Nrf} 2$ and activates the phosphorylation of Akt. The PI3k/Akt pathway is involved in SF-induced Nrf2 nuclear translocation, HO-1 expression and protection. SF may therefore serve as a useful cytoprotective agent in PD.

\section{Acknowledgements}

This study was supported by Science and Technology Jiont Special Fund of Yunnan Province (2009CD199).

\section{References}

1. Pakkenberg B, Moller A, Gundersen HJ, Mouritzen Dam A and Pakkenberg H: The absolute number of nerve cells in substantia nigra in normal subjects and in patients with Parkinson's disease estimated with an unbiased stereological method. J Neurol Neurosurg Psychiatry 54: 30-33, 1991.

2. Sian J, Dexter DT, Lees AJ, et al: Alterations in glutathione levels in Parkinson's disease and other neurodegenerative disorders affecting basal ganglia. Ann Neurol 36: 348-355, 1994. 
3. Castellani RJ, Perry G, Siedlak SL, et al: Hydroxynonenal adducts indicate a role for lipid peroxidation in neocortical and brainstem Lewy bodies in humans. Neurosci Lett 319: 25-28, 2002.

4. Hwang YP, Kim HG, Han EH and Jeong HG: Metallothionein-III protects against 6 -hydroxydopamine-induced oxidative stress by increasing expression of heme oxygenase-1 in a PI3K and ERK/ Nrf2-dependent manner. Toxicol Appl Pharmacol 231: 318-327, 2008

5. Blum D, Torch S, Lambeng N, et al: Molecular pathways involved in the neurotoxicity of 6-OHDA, dopamine and MPTP: contribution to the apoptotic theory in Parkinson's disease. Prog Neurobiol 65: 135-172, 2001.

6. Kienzl E, Puchinger L, Jellinger K, Linert W, Stachelberger H and Jameson RF: The role of transition metals in the pathogenesis of Parkinson's disease. J Neurol Sci 134 (Suppl): S69-S78, 1995.

7. Linert W, Herlinger E, Jameson RF, Kienzl E, Jellinger K and Youdim MB: Dopamine, 6-hydroxydopamine, iron, and dioxygen - their mutual interactions and possible implication in the development of Parkinson's disease. Biochim Biophys Acta 1316: $160-168,1996$.

8. Cohen G and Heikkila RE: The generation of hydrogen peroxide, superoxide radical, and hydroxyl radical by 6-hydroxydopamine, dialuric acid, and related cytotoxic agents. J Biol Chem 249 2447-2452, 1974

9. Hu X, Weng Z, Chu CT, et al: Peroxiredoxin-2 protects against 6-hydroxydopamine-induced dopaminergic neurodegeneration via attenuation of the apoptosis signal-regulating kinase (ASK1) signaling cascade. J Neurosci 31: 247-261, 2011.

10. Jakel RJ, Townsend JA, Kraft AD and Johnson JA: Nrf2-mediated protection against 6-hydroxydopamine. Brain Res 1144: 192-201, 2007.

11. Alam J and Cook JL: Transcriptional regulation of the heme oxygenase-1 gene via the stress response element pathway. Curr Pharm Des 9: 2499-2511, 2003.

12. Hung SY, Liou HC, Kang KH, Wu RM, Wen CC and Fu WM: Overexpression of heme oxygenase-1 protects dopaminergic neurons against 1-methyl-4-phenylpyridinium-induced neurotoxicity. Mol Pharmacol 74: 1564-1575, 2008.

13. Otterbein LE, Soares MP, Yamashita K and Bach FH: Heme oxygenase-1: unleashing the protective properties of heme. Trends Immunol 24: 449-455, 2003.

14. Stocker R, Yamamoto Y, McDonagh AF, Glazer AN and Ames BN: Bilirubin is an antioxidant of possible physiological importance. Science 235: 1043-1046, 1987.

15. Ramsey CP, Glass CA, Montgomery MB, et al: Expression of Nrf2 in neurodegenerative diseases. J Neuropathol Exp Neurol 66: $75-85,2007$

16. Itoh K, Tong KI and Yamamoto M: Molecular mechanism activating Nrf2-Keapl pathway in regulation of adaptive response to electrophiles. Free Radic Biol Med 36: 1208-1213, 2004.

17. Nakaso K, Yano H, Fukuhara Y, Takeshima T, Wada-Isoe K and Nakashima K: PI3K is a key molecule in the Nrf2-mediated regulation of antioxidative proteins by hemin in human neuroblastoma cells. FEBS Lett 546: 181-184, 2003.

18. Salinas M, Diaz R, Abraham NG, Ruiz de Galarreta CM and Cuadrado A: Nerve growth factor protects against 6-hydroxydopamine-induced oxidative stress by increasing expression of heme oxygenase-1 in a phosphatidylinositol 3-kinase-dependent manner. J Biol Chem 278: 13898-13904, 2003.

19. Zhang Y, Talalay P, Cho CG and Posner GH: A major inducer of anticarcinogenic protective enzymes from broccoli: isolation and elucidation of structure. Proc Natl Acad Sci USA 89: 2399-2403, 1992.
20. Mattson MP, Son TG and Camandola S: Viewpoint: mechanisms of action and therapeutic potential of neurohormetic phytochemicals. Dose Response 5: 174-186, 2007.

21. Ping Z, Liu W, Kang Z, et al: Sulforaphane protects brains against hypoxic-ischemic injury through induction of Nrf2-dependent phase 2 enzyme. Brain Res 1343: 178-185, 2010.

22. Jazwa A, Rojo AI, Innamorato NG, Hesse M, Fernandez-Ruiz J and Cuadrado A: Pharmacological targeting of the transcription factor nrf2 at the basal ganglia provides disease modifying therapy for experimental parkinsonism. Antioxid Redox Signal 14: 2347-2360, 2011

23. Tarozzi A, Morroni F, Merlicco A, et al: Sulforaphane as an inducer of glutathione prevents oxidative stress-induced cell death in a dopaminergic-like neuroblastoma cell line. J Neurochem 111: 1161-1171, 2009.

24. Han JM, Lee YJ, Lee SY, et al: Protective effect of sulforaphane against dopaminergic cell death. J Pharmacol Exp Ther 321: 249-256, 2007.

25. Soane L, Li Dai W, Fiskum G and Bambrick LL: Sulforaphane protects immature hippocampal neurons against death caused by exposure to hemin or to oxygen and glucose deprivation. J Neurosci Res 88: 1355-1363, 2010.

26. Tanito M, Masutani H, Kim YC, Nishikawa M, Ohira A and Yodoi J: Sulforaphane induces thioredoxin through the antioxidant-responsive element and attenuates retinal light damage in mice. Invest Ophthalmol Vis Sci 46: 979-987, 2005.

27. Itoh K, Ishii T, Wakabayashi N and Yamamoto M: Regulatory mechanisms of cellular response to oxidative stress. Free Radic Res 31: 319-324, 1999

28. Chen XL and Kunsch C: Induction of cytoprotective genes through Nrf2/antioxidant response element pathway: a new therapeutic approach for the treatment of inflammatory diseases. Curr Pharm Des 10: 879-891, 2004.

29. Chen K, Gunter K and Maines MD: Neurons overexpressing heme oxygenase-1 resist oxidative stress-mediated cell death. J Neurochem 75: 304-313, 2000.

30. Cuadrado A, Moreno-Murciano P and Pedraza-Chaverri J: The transcription factor Nrf2 as a new therapeutic target in Parkinson's disease. Expert Opin Ther Targets 13: 319-329, 2009.

31. Kensler TW, Wakabayashi N and Biswal S: Cell survival responses to environmental stresses via the Keap1-Nrf2-ARE pathway. Annu Rev Pharmacol Toxicol 47: 89-116, 2007.

32. Panahian N, Yoshiura M and Maines MD: Overexpression of heme oxygenase-1 is neuroprotective in a model of permanent middle cerebral artery occlusion in transgenic mice. J Neurochem 72: 1187-1203, 1999.

33. Zhao HD, Zhang F, Shen G, et al: Sulforaphane protects liver injury induced by intestinal ischemia reperfusion through Nrf2-ARE pathway. World J Gastroenterol 16: 3002-3010, 2010.

34. Hwang YP and Jeong HG: The coffee diterpene kahweol induces heme oxygenase-1 via the $\mathrm{PI} 3 \mathrm{~K}$ and $\mathrm{p} 38 / \mathrm{Nrf} 2$ pathway to protect human dopaminergic neurons from 6-hydroxydopamine-derived oxidative stress. FEBS Lett 582: 2655-2662, 2008

35. Hwang YP and Jeong HG: Ginsenoside Rb1 protects against 6-hydroxydopamine-induced oxidative stress by increasing heme oxygenase-1 expression through an estrogen receptor-related $\mathrm{PI} 3 \mathrm{~K} / \mathrm{Akt} / \mathrm{Nrf2}$-dependent pathway in human dopaminergic cells. Toxicol Appl Pharmacol 242: 18-28, 2010. 